[Agr. Biol. Chem,, Vol. 34, No. 6, p. 972 973, 1970]

\title{
A New Aromatic Amide, Caffeoylputrescine from Callus Tissue Culture of Nicotiana tabacum
}

Sir:

During tracer studies of the nicotine biosynthesis in callus tissue cultures of Nicotiana tabacum we observed that considerable amounts of the radioactivity from administered ${ }^{14} \mathrm{C}$ labelled putrescine or ornithine were incorporated into some ninhydrin-positive basic compounds. These compounds gave highly fluorescent spots under ultra-violet light on paper chromatograms.

In connection with our studies on the biosynthesis of nicotine, it seems to be of some importance and interest to identify such compounds. One of these was isolated and identified as 1-(3,4-dihydroxycinnamoylamino)4-aminobutane (caffeoylputrescine) from callus tissue cultures of $N$. tabacum.

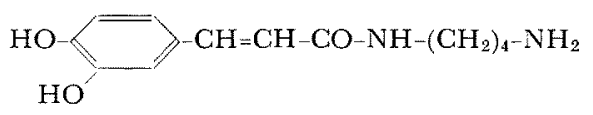

Five hundred $\mathrm{g}$ of the well washed tissue was repeatedly extracted with $75 \%$ aqueous ethanol. The extracts were passed through a column of Amberlite CG-50 resin in the hydrogen form. The column was washed with water and eluted with $4 \mathrm{~N}$ acetic acid. The eluate was concentrated to dryness under reduced pressure at $40^{\circ} \mathrm{C}$. The residue was dissolved in a small volume of water and subjected to cellulose column chromatography with $n$-butanol-acetic acid-water (65:10:25) as a solvent. The fractions containing the compound were dried and the residue was dissolved in a small volume of water. Repeated recrystallization from water afforded 110 $\mathrm{mg}$ of fine needles of the compound. The hydrochloride of the compound decomposed at $245 \sim 250^{\circ} \mathrm{C}$ with changing a form at about $96^{\circ} \mathrm{C}$. This change seems to be resulted from a loss of water of crystallization. Elementary analysis of the compound gave, $\mathrm{C}, 48.27 ; \mathrm{H}$, $7.00 ; \mathrm{N}, 8.78$ per cent. The calculated values for $\mathrm{C}_{13} \mathrm{H}_{18} \mathrm{~N}_{2} \mathrm{O}_{3} \cdot \mathrm{HCl} \cdot 2 \mathrm{H}_{2} \mathrm{O}$ were $\mathrm{C}, 48.28 ; \mathrm{H}$, $7.18 ; \mathrm{N}, 8.68$ per cent.

The compound gave violet color with ninhydrin and high fluorescence. The Le Rosen test for aromatic compounds, " Pauly's reaction ${ }^{2}$ and $\mathrm{FeCl}_{3}$ reaction were all positive. These results indicate that the presence of the amino group and phenolic moiety.

The UV absorption peaks of the compound in water are at $219,235,295$ and $320 \mathrm{~m} \mu$. The spectrum is closely similar to that of caffeic acid.

Hydrolysis of the compound by refluxing in $2 \mathrm{~N} \mathrm{NaOH}$ for $4 \mathrm{hr}$ under a nitrogen atmosphere gave caffeic acid and putrescine, which were identified by paper chromatography and infrared absorption spectroscopy.

The JR spectrum of the compound in $\mathrm{KBr}$ disk shows the usual feature for mono-substituted amides. The amide band A (carbonyl) is present at $1598 \mathrm{~cm}^{-1}$ and the amide band $B$ at $1536 \mathrm{~cm}^{-1}$ The absorption at $1655 \mathrm{~cm}^{-1}$ seems to be ascribed to the $\mathrm{C}=\mathrm{C}$ stretching vibration in conjugation with the carbonyl. The absorption at $980 \mathrm{~cm}^{-1}$ is considered to be due to the unsaturated $\mathrm{C}-\mathrm{H}$ deformation vibration at the trans-double bond. ${ }^{3}$

1) A. L. Le Rosen, R. T. Moravek and J. K. Cariton, Anal. Chem., 24, 1335 (1952).

2) T. Mann and E. Leone, Biochem. J., 53, 140 (1953).

3) L. Crombie, J. Chem. Soc., 1952, 2997. 
These results indicate that a possible structure of the compound to be 1-(3,4-dihydroxycinnamoylamino)-4-aminobutane. This is further confirmed by its NMR spectrum. The NMR spectrum of the compound in deuterium oxide shows the presence of three aromatic protons $(\delta$ 6.88, singlet and $\delta 6.76$, triplet formed by partial overlapping of two doublets) and two protons $(\delta 7.10$, doublet and $\delta 6.11$, doublet, $J$ value $15.0 \mathrm{c} / \mathrm{s}$ ) corresponding to vinyl protons. The signal of the vinyl proton which is adjacent to the carbonyl group shows a chemical shift at the lower field than the other proton because of the effects of the conjugated carbonyl group and the aromatic ring current. The coupling constant between the vinyl protons of $15.0 \mathrm{c} / \mathrm{s}$ confirmed the trans arrangement about the double bond." The spectrum also reveals four protons ( $\delta 3.05$ ) attributable to two methylene groups adjacent to amino groups and four additional protons ( $\delta$ 1.61) corresponding to the other methylene groups of 1,4-diaminobutane.

Final confirmation of the structure was obtained by comparison with synthetic 1-(3,4dihydroxycinnamoylamino)-4-aminobutane.

To a solution of excess putrescine in chloroform was added diacetylcaffeic acid chloride. Acetylcaffeoylputrescine thus obtained was separated by cellulose column chromatography with a solvent of $n$-butanol-acetic acid-water $(65: 10: 25)$. The acetyl derivative was treated with $2 \mathrm{~N} \mathrm{NaOH}$ and acidified with conc. $\mathrm{HCl}$ giving caffeoylputrescine hydrochloride (decomp. $245 \sim 250^{\circ} \mathrm{C}$ )

4) J. A. Pole, W. G. Schneider and H. J. Bernstein, "High-Resolution Nuclear Magnetic Resonance," McGraq-Hill Book Company Inc., 1956.
The IR, UV and NMR spectra of bot compounds were perfectly identical, $r$ spectively. These properties clearly show th the compound is 1-(3,4-dihydroxycinnamoy amino)-4-aminobutane.

Feruloylputrescine has been isolated ar identified from citrus leaves and fruits $k$ Wheaton and Stweart, ${ }^{51}$ and some relate aromatic amides such as aegelin, ${ }^{61}$ heraclavin cinnamoylhistamine, ${ }^{8}$ and p-coumaroylagm tine $^{9 /}$ have been found in plants. The occu rence of caffeoylputrescine in nature has $n$ previously been reported. Predominance cinnamic acid derivatives in tissue cultures tobacco plants are notable. Bergmann et al. observed the occurrence of the glucosamis ester of hydroxycinnamic acid derivatives tissue cultures of $N$. tabacum.

Further work is now under way to stur its physiological role in the cells and inves gate its possible role in the biosynthesis nicotine.

Shigenobu MizUSA

Yoko TANABE

Masao NOGUCHI

The Central Research Institute,

Japan Monopoly Corporation,

Nishishinagawa, Shinagawa-ku, Tokyo

Received March 17, 19

5) T. A. Wheaton and I. Stweart, Nature, 206, 6 (1965).

6) R. N. Chakravarti and B. Dasgupta, J. Che Soc., 1958, 1580 .

7) L. Crombie, ibid., 1955, 995.

8) J. S. Fitzerald, Australian J. Chem., 17, 3 (1964).

9) A. Stossel, Phytochem., 4, 973 (1965).

10) L. Bergmann, W. Thies and K. Erdelsky, Naturforsch., 20, 1297 (1965). 